\title{
Batata-doce biofortificada como alternativa no combate à deficiência de vitamina A em crianças
}

\author{
Biofortified sweet potatoes as an alternative to fight against \\ vitamin A deficiency in children
}

Gilberto Arcanjo Fagundes ${ }^{1}$

Nadine Soares Pinto²

RECEBIDO EM 24/03/2021

Adriana Aparecida Hansel Michelotti ${ }^{3}$

Melissa Walter ${ }^{4}$

Joseana Severo ${ }^{5}$

\begin{abstract}
RESUMO
A fome oculta ocorre quando o consumo e/ou absorção de micronutrientes é insuficiente para sustentar o correto desenvolvimento. A carência de vitamina A pode causar deficiência visual, aumento do risco de desenvolvimento de doenças graves e morte em crianças. O $\beta$-caroteno (precursor da vitamina A) é um carotenoide presente em cultivares de batata-doce biofortificadas (BDB), que apresentam polpa alaranjada. Foram investigados materiais de domínio público, em bases de dados digitais, buscando assuntos relacionados à deficiência de vitamina A em crianças e à ingestão diária recomendada (IDR), assim como produtos alimentícios obtidos a partir de BDB e teor de $\beta$-caroteno em
\end{abstract}

1 Doutor na Universidade Federal do Rio Grande, FURG, Rio Grande do Sul, Brasil. arcanjogaf@yahoo.com.br - https://orcid.org/0000-0002-7761-8365

2 Discente do curso de Especialização em Gestão da Qualidade e Novas Tendências em Alimentos, Instituto Federal Farroupilha, IFFar, Campus Santa Rosa, Rio Grande do Sul, Brasil. snadine184@gmail.com - https://orcid.org/0000-0002-6018-5233

3 Professora Mestre no Instituto Federal Farroupilha, IFFar, Campus Santa Rosa, Rio Grande do Sul, Brasil. adriana.michelotti@iffarroupilha.edu.br - https://orcid.org/0000-0002-7537-0140

4 Professora Doutora no Instituto Federal Farroupilha, IFFar, Campus Santa Rosa, Rio Grande do Sul, Brasil. melissa.walter@iffarroupilha.edu.br - https://orcid.org/0000-0003-0535-0352

5 Professora Doutora no Instituto Federal Farroupilha, IFFar, Campus Santa Rosa, Rio Grande do Sul, Brasil. joseana.severo@iffarroupilha.edu.br - https://orcid.org/0000-0003-0571-1955 
variedades e produtos elaborados com batatas cultivadas no Brasil. A partir dos dados obtidos foram apresentados: as condições de processamento, concentração de $\beta$-caroteno e quantidade necessária para suprir a IDR de vitamina A em crianças de cada produto (batata-doce ou alimento) avaliado. Foram tabelados vinte produtos, cujos teores de $\beta$-caroteno variaram entre 0,17 e 3,79 mg $\beta$-caroteno/100 g, sendo necessária uma porção de 65 g do cultivar da BDB para suprir a IDR (400 $\mu \mathrm{g}$ RE/dia) de vitamina A de crianças de até seis anos. O consumo dos produtos alimentícios preparados com BDB variaram entre $830 \mathrm{~g}$ e $71 \mathrm{~g}$, demonstrando que a forma de preparo, como o uso do calor aliado à exposição ao $\mathrm{O}_{2}$, pode resultar em degradação dos carotenoides. O desenvolvimento de produtos alimentícios com altos teores de $\beta$-caroteno apresenta-se como uma alternativa viável para a inserção do BDB. As condições de consumo também devem ser levadas em consideração, visando a melhorar a bioacessibilidade e a biodisponibilidade e a combater a deficiência de vitamina A.

PALAVRAS-CHAVE: fome oculta; biofortificação; processamento alimentício; $\beta$-caroteno.

\begin{abstract}
Hidden hunger occurs when the consumption and/or absorption of micronutrients is insufficient to support proper development. Vitamin A deficiency can cause visual impairment, as well as an increased risk of developing serious illnesses and death to children. $\beta$-carotene (precursor to vitamin $A$ ) is a carotenoid present in biofortified sweet potato (BDB) cultivars, which have orange flesh. Public domain materials were investigated in digital databases, searching for issues related to vitamin A deficiency in children and the recommended daily intake (IDR), as well as food products obtained from BDB and $\beta$-carotene content in different varieties produced in Brazil and in products made from sweet potatoes. The processing conditions, $\beta$-carotene concentration, and the necessary amount to supply the vitamin A RDI for children of each product (food product or sweet potato) were presented. Twenty food products were presented, whose levels of $\beta$-carotene varied from 0.17 to $3.79 \mathrm{mg} \beta$-carotene $/ 100 \mathrm{~g}$. A portion of $65 \mathrm{~g}$ of BDB is required to supply the vitamin A RDI ( $400 \mu \mathrm{g}$ RE/day) for children up to six years old. Among the food products added with BDB, this portion ranges from $71 \mathrm{~g}$ and $830 \mathrm{~g}$ to supply the same amount of vitamin A RDI for children. Also, the form of preparation, such as the heat treatment, combined with exposure to $\mathrm{O}_{2}$ enhances carotenoid degradation. The development of food products containing high levels
\end{abstract}


of $\beta$-carotene presents a viable alternative for the insertion of BDB. Consumption conditions, aiming to improve bioaccessibility and bioavailability, must also be taken into account, seeking to fight against vitamin A deficiency.

KEYWORDS: hidden hunger; biofortification; food processing; $\beta$-carotene.

\section{Introdução}

Segundo a Organização das Nações Unidas para Agricultura e Alimentação (FAO, 2014), a fome é geralmente entendida como uma aflição associada à falta de comida e/ou à privação alimentar e/ou subnutrição (consumo menor do que $1.800 \mathrm{kcal}$ por dia - o mínimo que a maioria das pessoas precisa para ter uma vida saudável e produtiva). A desnutrição (resultado da ingestão inadequada de alimentos - em quantidade ou qualidade) e as doenças crônicas relacionadas à dieta são as principais causas de morte no mundo, e, todos os anos, milhões de pessoas perdem a vida em decorrência de deficiências nutricionais (FAO, 2014).

A fome oculta ocorre quando o consumo ou a absorção de micronutrientes (vitaminas e minerais) não é suficiente para sustentar a boa saúde e o desenvolvimento em crianças e as funções físicas e mentais normais em adultos (IFPRI, 2014). Estima-se que 2 bilhões de pessoas no mundo sofram de carência de micronutrientes; em muitos desses casos, porém, não são identificados sinais tipicamente associados à fome e à desnutrição (SALTZMAN et al., 2016; BOUIS; SALTZMAN, 2017; SEVERO et al., 2021).

A deficiência de micronutriente mais significativa do ponto de vista da saúde pública é a de vitamina A, com uma estimativa de 190 milhões de crianças em idade pré-escolar e 19 milhões de mulheres grávidas afetadas (FAO, 2014). A deficiência de vitamina A em crianças em idade pré-escolar pode causar grave deficiência visual, que leva à cegueira, aumento do risco de doenças graves e morte por infecções comuns, como diarreia e sarampo. 
Em mulheres grávidas, pode causar cegueira noturna e aumento do risco de morte (FAO, 2014; IFPRI, 2014).

A suplementação de vitamina A é uma das intervenções para melhorar a sobrevivência infantil (TAN-TORRES et al., 2005). Os programas para suplementar a vitamina A estão muitas vezes integrados nas políticas nacionais de saúde, porque estão associados à redução na mortalidade infantil e a uma menor incidência de diarreia (IMDAD et al., 2010). Segundo a UNICEF, pelo menos 70\% das crianças de seis a sessenta meses precisam receber suplementos de vitamina A a cada seis meses para que se obtenham as reduções desejadas na mortalidade infantil (FAO, 2014).

Os métodos de processamento apresentam forte influência sobre a composição nutricional dos alimentos, podendo resultar em degradação de importantes nutrientes, como as vitaminas. As vitaminas, dependendo das suas características, podem ser degradadas por diferentes fatores, como temperatura, presença de oxigênio, luminosidade, pH, duração do método de processamento, entre outros (CORREIA; FARAONI; PINHEIRO-SANTANA, 2008).

A biofortificação de espécies tem sido uma ferramenta importante no aumento do teor de micronutrientes essenciais para saúde humana e, consequentemente, no combate à desnutrição, especialmente nas populações mais pobres (SEVERO et al., 2021). No Brasil, visando a contribuir com o suprimento de vitamina A por meio da alimentação, a Empresa Brasileira de Pesquisa Agropecuária (Embrapa) tem selecionado e trabalhado no melhoramento de culturas pela biofortificação convencional, que não utiliza transgenia, como é o caso da batata-doce biofortificada, que apresenta maiores teores de $\beta$-caroteno, um precursor da vitamina $A$.

Dessa forma, realizou-se uma pesquisa bibliográfica com o intuito de elencar a batata-doce biofortificada como uma ferramenta de combate à hipovitaminose A em crianças. Por meio da pesquisa bibliográfica sobre o 
uso de batata-doce biofortificada em preparos alimentícios, foram calculadas as quantidades necessárias de vitamina A para suprir a IDR (índice diário recomendado) em crianças.

\section{Referencial teórico}

\subsection{Batata-doce (Ipomoea batatas Lam.)}

A batata-doce está entre as sete culturas agrícolas básicas mais importantes, sendo cultivada em mais de cem países. É uma hortaliça tuberosa rústica, de fácil e baixo custo de produção, com versatilidade para se adaptar a diferentes tipos de solo e clima (LAURIE et al. 2015; CAVALCANTE et al., 2006).

No Brasil, a batata-doce ocupa o sexto lugar entre as hortaliças mais plantadas, com uma produção de 805.412 toneladas em 2019, em uma área estimada de 57.290 hectares (FAOSTAT, 2021). O Brasil se destaca por apresentar uma alta diversidade genética de batata-doce, que se traduz em raízes de várias formas, tamanhos e cores (AZEVEDO et al. 2015). Além disso, a batata-doce é um alimento interessante do ponto de vista nutricional, por ser fonte de vitaminas e minerais (VIZZOTTO et al., 2018), sendo uma importante fonte de alimentação para as populações carentes.

Devido à sua composição, a batata-doce é uma boa alternativa como fonte de nutrientes e energia (AZEVEDO et al., 2015). Na alimentação humana, pode ser consumida assada, cozida, frita ou na forma de farinha; os animais, por sua vez, podem se alimentar tanto de suas ramas quanto das raízes, frescas ou na forma de silagem (ANDRADE Jr. et al., 2012; VIANA et al., 2011; FIGUEIREDO et al., 2012).

Além de vitaminas e minerais, cultivares de batata-doce de polpa alaranjada apresentam, em sua composição, altos níveis de carotenoides, com destaque para o $\beta$-caroteno, um precursor da vitamina A. Os carotenoides também são 
reconhecidos pela sua atividade antioxidante e outros benefícios que trazem à saúde humana, como melhoria da imunidade e diminuição de doenças degenerativas, doenças cardiovasculares e câncer (VAN JAARSVELD et al., 2006; BOVELL-BENJAMIN, 2007; RODRIGUEZ-AMAYA et al., 2008; COSTA, ROSA, 2010; VIZZOTTO et al., 2017).

O $\beta$-caroteno é o principal carotenoide relatado em cultivares de batata-doce de polpa alaranjada, sendo atualmente a cultivar Beauregard uma das que apresentam maiores teores superiores de $\beta$-caroteno (SUAREZ et al., 2016). De acordo com dados da Embrapa (2021), em variedades de polpa branca, a concentração de betacaroteno é inferior a 10 mg/g de raiz. Na cultivar Beauregard, o teor pode chegar a $115 \mu \mathrm{g} / \mathrm{g}$ de raiz; por isso, ela é considerada uma batata-doce biofortificada. Failla, Thakkar e Kim (2009), analisando oito cultivares de batata-doce de polpa laranja cultivadas em outros países, identificaram unicamente o isômero alltrans $\beta$-caroteno nesses clones, e observaram teores variando de 112 a $281 \mu \mathrm{g} / \mathrm{g}$.

\subsection{Desnutrição infantil e a carência de vitamina A}

A desnutrição é o resultado da ingestão inadequada de alimentos (em termos de quantidade ou qualidade). Segundo a Organização das Nações Unidas para Agricultura e Alimentação (FAO, 2014), a fome é geralmente entendida como uma aflição associada à falta de comida, privação alimentar ou subnutrição. Em geral, quando se trata de calorias necessárias, ocorrem variações de acordo com a faixa etária das crianças: recém-nascidos requerem de $100 \mathrm{kcal} / \mathrm{kg} /$ dia a $150 \mathrm{kcal} / \mathrm{kg} /$ dia; lactentes, de 100 a $140 \mathrm{kcal} / \mathrm{kg} /$ dia; pré-escolares (1 - 7 anos), de 75 a 90 kcal/kg/dia; escolares (7 - 12 anos), de 60 a 75 kcal/kg/dia; e adolescentes (12 - 18 anos), de 30 a 60 kcal/kg/dia (SPOLIDORO, 2000). 
A desnutrição causada por deficiência de micronutrientes (vitaminas e minerais) é normalmente conhecida por "fome oculta", porque a maioria das pessoas afetadas não mostra sinais tipicamente associados à fome e desnutrição. Isso ocorre quando o consumo ou absorção de micronutrientes é muito baixa para sustentar a boa saúde e o desenvolvimento de crianças e as funções físicas e mentais normais em adultos (IFPRI, 2014). Portanto, assim como a desnutrição, a deficiência de micronutrientes ou fome oculta é uma violação do direito de uma criança a um padrão de vida adequado para o desenvolvimento físico e mental (FAO, 2014).

O número de pessoas acometidas por desnutrição é, geralmente, obtido por meio da quantidade de calorias consumidas pela população, e não pelos índices de ingestão de micronutrientes pelos grupos vulneráveis, como crianças e mulheres. A carência desses micronutrientes pode desencadear diversos problemas de saúde, como a anemia, condição que causa fraqueza, cansaço e tontura e dificulta o percurso educacional e a interação social de crianças. Projeta-se que $42 \%$ das crianças de até cinco anos de todo o mundo sejam anêmicas e que 15,4\% das crianças com menos de 5 anos tenham essa deficiência. A insuficiência de vitamina A é uma das causas da cegueira em crianças e do aumento do risco de doenças e infecções graves. Mulheres grávidas são mais suscetíveis à deficiência de vitamina A, principalmente no fim da gravidez. Estima-se que 19 milhões de mulheres grávidas tenham deficiência de vitamina A (FAO, 2014). Já no início da vida, a melhor forma de proteger os bebês dessa carência nutricional é a amamentação materna, visto que a suplementação de vitamina A para crianças é indicada a partir dos seis meses de idade (WHO, 2021).

A deficiência em vitamina A é a mais significativa do ponto de vista da saúde pública. Estima-se que atinja 190 milhões de crianças em idade pré-escolar (FAO, 2014) e 17,4\% das crianças com menos de cinco anos no Brasil. 
A situação é mais grave nas regiões Sudoeste e Nordeste do país, com índices de 22,1\% e 19\%, respectivamente (LIMA; DAMIANI; FUMIMORI, 2018).

Estudo divulgado pela FAO indica uma alta correlação entre a baixa diversidade alimentar e a deficiência de vitamina A e a taxa de mortalidade em crianças com menos de cinco anos. Além disso, a mortalidade antes dos cinco anos de idade apresenta uma forte correlação com a incidência de anemia em crianças e mulheres grávidas e com a cegueira noturna em crianças em idade pré-escolar e mulheres grávidas (FAO, 2014).

Os programas para suplementar a vitamina A estão muitas vezes integrados às políticas nacionais de saúde, porque estão associados à redução da mortalidade infantil e a uma incidência reduzida de diarreia (IMDAD et al., 2010), sendo a suplementação de vitamina A uma das abordagens para reduzir a mortalidade infantil (TAN-TORRES et al., 2005). Segundo a UNICEF, para se alcançar as reduções desejadas na mortalidade infantil, pelo menos 70\% das crianças de seis a sessenta meses precisam receber suplementos de vitamina A semestralmente (FAO, 2014).

Podemos ingerir vitamina A pré-formada a partir de alimentos de origem animal, sendo a carne de fígado e o óleo de pescado as melhores fontes. Contudo, estas fontes são caras para um consumo regular pela maioria da população mundial. Nos países com menor desenvolvimento, aproximadamente $82 \%$ do total de vitamina A ingerida é obtida de carotenoides. O $\beta$-caroteno é o carotenoide mais comum nas fontes alimentares; ele é encontrado em diversos vegetais de coloração verde e laranja (cenoura, batata-doce de cor laranja, manga, abóbora) (BURRI, 2011).

\subsection{A biofortificação e a segurança alimentar e nutricional}

A biofortificação envolve o aprimoramento de culturas alimentares, por meio de diferentes métodos, para aumentar o conteúdo de micronutrientes 
e melhorar o rendimento e a resistência a pragas, bem como características de consumo, como sabor e tempo de cozimento (FAO, 2014). O incremento nutricional pode ser realizado de maneira convencional, executando-se repetidos cruzamentos; por transgenia, processo conhecido como biofortificação transgênica; ou, ainda, pelo manejo da planta no campo, especialmente a partir da adubação (ZHU et al., 2007).

Produtos biofortificados via melhoramento convencional vêm sendo desenvolvidos no Brasil e em países da América Central, da África e da Ásia, para combater a "fome oculta" (NESTEL et al., 2006). Apesar dos esforços para contê-la, com o uso de suplementos e alimentos fortificados, a deficiência ainda é, entre as de caráter nutricional, a mais comum no mundo, afetando 1,6 bilhão de pessoas (BIOFORT, 2021).

A base do melhoramento de uma cultura agrícola está em sua variabilidade genética, ou seja, na diversidade de resposta às melhores práticas agronômicas, resistência à pragas e doenças, tolerância à seca, etc. (CARDOSO et al., 2005). A biofortificação convencional surgiu com o HarvestPlus, programa global focado em segurança nutricional que vem desenvolvendo cultivares biofortificadas com o objetivo de reduzir a fome oculta no mundo (BURRI, 2011).

No Brasil, os projetos de biofortificação de alimentos são coordenados pela Embrapa, através da Rede BioFORT. Cultivares de mandioca, feijão, arroz e batata-doce enriquecidas com ferro (Fe), zinco (Zn) e $\beta$-caroteno já foram identificadas e estão sendo introduzidas nas pequenas propriedades rurais, em ações conjuntas da Embrapa, Emater, universidades e outras instituições (BIOFORT, 2021).

Devido à sua elevada rusticidade e amplo espectro de potencialidade de uso, a batata-doce pode ser considerada uma espécie de interesse econômico, principalmente para países em desenvolvimento e com escassez de alimentos (CARDOSO et al., 2005). 
O consumo de alimentos de alta qualidade nutricional é uma das melhores estratégias para combater as carências nutricionais, como a hipovitaminose A. Essas carências afetam numerosos grupos populacionais, fato que demanda pesquisas para elucidação da composição química de vegetais de baixo custo, cuja produção seja sustentável, para contribuir com o tratamento de indivíduos acometidos por essas desordens nutricionais (NASCIMENTO et al., 2013).

Nascimento et al. (2013) afirmam que a fécula de batata-doce tem um elevado potencial de utilização na biofortificação de alimentos para a merenda escolar, contribuindo, também, com o fortalecimento da agricultura familiar orgânica. Ações nas quais foram distribuídas variedades de batata-doce biofortificadas melhoraram significativamente o consumo de vitamina A de mulheres e crianças (HOTZ et al., 2012a; HOTZ et al., 2012b).

\section{Metodologia}

Foram pesquisados em materiais de domínio público, disponíveis preferencialmente em bases de dados digitais (Periódicos da Capes, Google Acadêmico, etc.), sem delimitação de tempo, os seguintes assuntos: a) hipovitaminose A em crianças; b) necessidades de ingestão diária de vitamina A por crianças; c) biofortificação de batatas-doces; d) obtenção de produtos alimentícios a partir de batatas-doces biofortificadas; e e) teor de $\beta$-caroteno e/ou vitamina A em variedades de batatas-doces convencionais e biofortificadas e em produtos cultivados e desenvolvidos no Brasil. Com base nos assuntos pesquisados, foi criada uma tabela contendo: a) cultivares de batata-doce e produtos alimentícios obtidos a partir delas; b) condições a que foram submetidas e/ ou processadas; c) concentração de $\beta$-caroteno presente nas cultivares de batata-doce/produto; d) porção necessária dos respectivos produtos para suprir a ingestão diária recomendada (IDR) de vitamina A para crianças de zero a seis anos de idade; e e) a referência do estudo. 


\section{O consumo de $\beta$-caroteno no combate à hipovitaminose $A$}

Conforme o Quadro 1, obtido do estudo realizado por Burri (2011), as crianças de até seis anos de idade necessitam uma média aproximada de $400 \mu \mathrm{g}$ de retinol equivalente (RE) ao dia, o que representa $2.400 \mu \mathrm{g}(2,4 \mathrm{mg})$ de $\beta$-caroteno. De acordo com a FAO (2004), estima-se que 6 ug de $\beta$-caroteno são equivalentes a 1 g de vitamina A (1 RE), na conversão.

\section{QUADRO 1 - Ingestão diária recomendada de vitamina A segundo a WHO/FAO (2004).}

\begin{tabular}{|lc|}
\hline Faixa etária & IDR $(\mu \mathrm{g}$ RE) \\
\hline crianças 0 a 6 meses & 375 \\
\hline crianças 7 a 12 meses & 400 \\
\hline crianças 1 a 3 anos & 400 \\
\hline crianças 4 a 6 anos & 450 \\
\hline crianças 7 a 8 anos & 500 \\
\hline crianças 9 anos & 500 \\
\hline crianças 10 a 13 anos & 600 \\
\hline adolescentes 14 a 18 anos & 600 \\
\hline
\end{tabular}

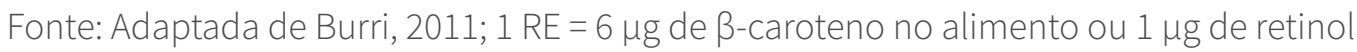
purificado. RE (retinol equivalente) é a unidade de medida usada pela WHO para descrever a quantidade de vitamina A fornecida nos alimentos pelos carotenoides.

A taxa de conversão de $\beta$-caroteno em vitamina A depende de muitos fatores, entre os quais o tipo de carotenoide, da matriz alimentícia e do próprio indivíduo. É sabido, por exemplo, que a absorção de $\beta$-caroteno é maior na presença de lipídios (BURRI, 2011).

Na Tabela 1 estão dispostos os dados de vinte produtos/cultivares de batata-doce, resultantes de estudos que desenvolveram diferentes produtos a partir de cultivares de batata-doce, que poderiam ser incorporados à alimentação infantil. Apresentam-se suas respectivas condições de processamento, teor de $\beta$-caroteno e porção necessária para suprir a necessidade diária de vitamina A em crianças de zero a seis anos de idade. Utilizou-se, 
como referência, a média dos valores do Quadro 1 (400 RE de $\beta$-caroteno = 2,4 mg de $\beta$-caroteno).

TABELA 1 - Teor de $\beta$-caroteno em diferentes cultivares de batata-doce e/ou seus produtos e porção necessária para suprir a IDR de crianças de até seis anos.

\begin{tabular}{|c|c|c|c|c|}
\hline Produto/cultivar & $\begin{array}{l}\text { Condições de } \\
\text { processamento }\end{array}$ & $\begin{array}{c}{[\beta \text {-caroteno }} \\
\mathrm{mg} / 100 \mathrm{~g}]\end{array}$ & $\begin{array}{c}\text { Porção } \\
\text { necessária } \\
\text { produto* }\end{array}$ & Referência \\
\hline $\begin{array}{l}\text { Farinha de BD } \\
\text { Brazlândia Roxa }\end{array}$ & Secagem $40^{\circ} \mathrm{C} / 24 \mathrm{~h}$ & 0,25 & $1 \mathrm{~kg}$ & $\begin{array}{l}\text { Remonato et } \\
\text { al. (2017) }\end{array}$ \\
\hline $\begin{array}{l}\text { Farinha de BDB } \\
\text { Beauregard }\end{array}$ & Secagem $40^{\circ} \mathrm{C} / 24 \mathrm{~h}$ & 2,32 & $100 \mathrm{~g}$ & $\begin{array}{l}\text { Remonato et } \\
\text { al. (2017) }\end{array}$ \\
\hline $\begin{array}{l}\text { Macarrão "talharim" com 20\% } \\
\text { de farinha de BDB Beauregard }\end{array}$ & Secagem $40^{\circ} \mathrm{C} / 24 \mathrm{~h}$ & 0,89 & $270 \mathrm{~g}$ & $\begin{array}{l}\text { Remonato et } \\
\text { al. (2017) }\end{array}$ \\
\hline BDB cv. Beauregard & Assada $200^{\circ} \mathrm{C} / 40 \mathrm{~min}$ & 3,79 & $65 \mathrm{~g}$ & $\begin{array}{l}\text { Santos et al. } \\
(2018)\end{array}$ \\
\hline BDB polpa alaranjada & Crua & 3,0 & $80 \mathrm{~g}$ & $\begin{array}{l}\text { Santos et al. } \\
(2018)\end{array}$ \\
\hline BDB polpa alaranjada & $\begin{array}{l}\text { Cozida } \\
100^{\circ} \mathrm{C} / 40 \mathrm{~min}\end{array}$ & 2,51 & $95 \mathrm{~g}$ & $\begin{array}{l}\text { Santos et al. } \\
(2018)\end{array}$ \\
\hline BD polpa roxa & Crua & 0,7 & $345 \mathrm{~g}$ & $\begin{array}{l}\text { Santos et al. } \\
(2018)\end{array}$ \\
\hline BD polpa roxa & $\begin{array}{l}\text { Cozida } \\
100^{\circ} \mathrm{C} / 40 \mathrm{~min}\end{array}$ & 0,21 & $1,15 \mathrm{~kg}$ & $\begin{array}{l}\text { Santos et al. } \\
(2018)\end{array}$ \\
\hline BDB BRS Amélia & Crua & 0,175 & $1,37 \mathrm{~kg}$ & $\begin{array}{l}\text { Pletsch et al. } \\
\text { (2016) }\end{array}$ \\
\hline BDB BRS Amélia & Cozida $40 \mathrm{~min}$ & 0,17 & $1,41 \mathrm{~kg}$ & $\begin{array}{l}\text { Pletsch et al. } \\
\text { (2016) }\end{array}$ \\
\hline Pão c/ 25\% de BDB Beauregard & $\begin{array}{l}\text { Assado } \\
160^{\circ} \mathrm{C} / 30 \mathrm{~min}\end{array}$ & 1,09 & $220 \mathrm{~g}$ & $\begin{array}{l}\text { Santos et al. } \\
(2021)\end{array}$ \\
\hline $\begin{array}{l}\text { Doce de cacau feito c/ } 54 \% \\
\text { BDB Beauregard }\end{array}$ & Cozimento por $1520 \mathrm{~min}$ & 0,92 & $260 \mathrm{~g}$ & $\begin{array}{l}\text { Santos et al. } \\
(2021)\end{array}$ \\
\hline $\begin{array}{l}\text { Doce de coco feito com 57\% } \\
\text { BDB Beauregard }\end{array}$ & Cozimento por $1520 \mathrm{~min}$ & 1,91 & $125 \mathrm{~g}$ & $\begin{array}{l}\text { Santos et al. } \\
(2021)\end{array}$ \\
\hline $\begin{array}{l}\text { Bolo com 31\% de BDB } \\
\text { Beauregard }\end{array}$ & $\begin{array}{l}\text { Cozimento da BDB } \\
\left(40 \mathrm{~min} / 100^{\circ} \mathrm{C}\right) \text { e do bolo } \\
\left(180^{\circ} \mathrm{C} / 30 \mathrm{~min}\right)\end{array}$ & 3,09 & $78 \mathrm{~g}$ & $\begin{array}{l}\text { Santos et al. } \\
(2021)\end{array}$ \\
\hline $\begin{array}{l}\text { Sorvete com } 40 \% \text { de BDB } \\
\text { Beauregard }\end{array}$ & $\begin{array}{l}\text { Cozimento da BDB } \\
\left(40 \mathrm{~min} / 100^{\circ} \mathrm{C}\right)\end{array}$ & 3,4 & $71 \mathrm{~g}$ & $\begin{array}{l}\text { Santos et al. } \\
(2018)\end{array}$ \\
\hline $\begin{array}{l}\text { Bala com } 40 \% \text { de BDB Amélia } \\
\text { (sabor morango) }\end{array}$ & $\begin{array}{l}\text { Cozimento da BDB (40 min) } \\
\text { e da bala ( } 20 \mathrm{~min})\end{array}$ & 0,07 & $365 \mathrm{~g}$ & $\begin{array}{l}\text { Pletsch et al. } \\
(2016)\end{array}$ \\
\hline $\begin{array}{l}\text { Bala com 40\% de BDB Amélia } \\
\text { (sem sabor) }\end{array}$ & $\begin{array}{l}\text { Cozimento da BDB (40 min) } \\
\text { e da bala ( } 20 \mathrm{~min})\end{array}$ & 0,03 & $830 \mathrm{~g}$ & $\begin{array}{l}\text { Pletsch et al. } \\
\text { (2016) }\end{array}$ \\
\hline $\begin{array}{l}\text { Bala com 40\% de BDB Amélia } \\
\text { (sabor morango) }\end{array}$ & $\begin{array}{l}\text { Cozimento da BDB (40 min) } \\
\text { e da bala ( } 20 \mathrm{~min})\end{array}$ & 0,07 & $350 \mathrm{~g}$ & $\begin{array}{l}\text { Pletsch et al. } \\
\text { (2016) }\end{array}$ \\
\hline
\end{tabular}




\begin{tabular}{|c|c|c|c|c|}
\hline Produto/cultivar & $\begin{array}{l}\text { Condições de } \\
\text { processamento }\end{array}$ & $\begin{array}{c}{[\beta \text {-caroteno }} \\
\mathrm{mg} / 100 \mathrm{~g}]\end{array}$ & $\begin{array}{l}\text { Porção } \\
\text { necessária } \\
\text { produto* }^{*}\end{array}$ & Referência \\
\hline $\begin{array}{l}\text { Bala com } 40 \% \text { de BDB Amélia } \\
\text { (sem sabor) }\end{array}$ & $\begin{array}{l}\text { Cozimento da BDB (40 min) } \\
\text { e da bala ( } 20 \mathrm{~min})\end{array}$ & 0,10 & $230 \mathrm{~g}$ & $\begin{array}{l}\text { Pletsch et al. } \\
(2016)\end{array}$ \\
\hline Fécula BDB alaranjada & $\begin{array}{l}\text { cozimento brando por } \\
20 \mathrm{~min}+\text { secagem a } 65^{\circ} \mathrm{C} \\
\text { por } 25 \mathrm{~h}\end{array}$ & 18 & $13 \mathrm{~g}$ & $\begin{array}{l}\text { Nascimento et } \\
\text { al. (2013) }\end{array}$ \\
\hline
\end{tabular}

$\mathrm{BD}=$ batata-doce; $\mathrm{BDB}=$ batata-doce biofortificada; ${ }^{*}$ Conversão: $1 \mathrm{RE}=6 \mu \mathrm{g}$ de $\beta$-caroteno no alimento ou 1 ug de retinol purificado (WHO/FAO, 2004).

É possível observar na Tabela 1 que, além do tipo de cultivar (convencional ou biofortificada), o processo de cocção da batata-doce e dos produtos formulados a partir dela provoca a redução do teor de carotenoides, esta variando conforme as condições aplicadas.

Das cultivares e/ou produtos avaliados (Tabela 1), a batata-doce Beauregard, assada a $200^{\circ} \mathrm{C}$ por 40 minutos, foi a fonte que apresentou maior teor de $\beta$-caroteno (3,79 mg/100 g). Considerando a IDR recomendada pela WHO/ FAO (2004), seriam necessários 65 g desse produto para suprir a necessidade de vitamina A em crianças de até seis anos. Fernandes et al. (2014) estimam que o consumo de 25 a $50 \mathrm{~g}$ de batata-doce cv. Beauregard supriria a IDR de vitamina A recomendada para adultos.

Em contrapartida, a variedade de BDB BRS Amélia, quando submetida ao cozimento por 40 minutos, foi a fonte que apresentou a menor concentração de $\beta$-caroteno (0,17 mg/100 g) (Pletsch et al. 2016), e, portanto, seria necessário 1,41 kg desse produto para suprir a IDR de vitamina A em crianças de até seis anos, quantidade 22 vezes maior que a da variedade biofortificada Beauregard. A variedade BDB BRS Amélia é uma cultivar que apresenta teores superiores de $\beta$-caroteno, em comparação com as cultivares convencionais; no entanto, apresenta teores inferiores aos da variedade BDB Beauregard (VIZZOTO, et al., 2017)

Muitos estudos têm buscado melhorar o valor nutritivo de produtos alimentícios pelo uso de batatas-doces biofortificadas, despertando a atenção dos 
consumidores que buscam uma alimentação mais saudável. No entanto, a apresentação e a aceitação de novas cultivares e de produtos obtidos a partir delas é um desafio para os pesquisadores, pois os consumidores têm perfis sensoriais diferentes (LAURIE et al., 2015).

Os carotenoides são facilmente degradados por estímulos ambientais (em particular o oxigênio atmosférico) durante o processamento e estocagem, e perdas decorrentes de danos físicos, como descascamento, corte e oxidações também podem ocorrer (RODRIGUEZ-AMAYA, 1999; RODRIGUEZ-AMAYA et al., 2008).

De acordo com Vizzoto et al. (2017), cultivares de batata-doce de polpa alaranjada, como a Beauregard, podem apresentar dez vezes mais carotenoides totais se comparados com cultivares de polpa branca ou creme. O $\beta$-caroteno é o principal carotenoide relatado em cultivares de polpa alaranjada.

Conforme Burri (2011), variedades de produtos alimentícios naturalmente ricos em $\beta$-caroteno (batata-doce de polpa alaranjada) têm sido utilizados com sucesso em intervenções de pequena escala para aumentar os níveis de vitamina A. Embora a batata-doce de polpa alaranjada contenha grande quantidade de $\beta$-caroteno, a sua totalidade não está acessível. A bioacessibilidade é definida como a fração de carotenoides transferidos do alimento para as micelas mistas do intestino, que fica, portanto, acessível para a subsequente absorção pela mucosa intestinal (BURRI, 2011).

Estudos envolvendo o metabolismo de carotenoides sugerem que essa baixa absorção dos carotenoides proveniente dos alimentos é a principal razão da baixa taxa de conversão de $\beta$-caroteno em vitamina A. A bioacessibilidade dos carotenoides depende da matriz alimentícia, do tipo de fibra e de gordura do alimento e do aquecimento e homogeneização no processamento do alimento (VEDA et al., 2006; BENGTSSON; ALMINGER; SVANBERG, 2009; MILLS et al., 2009). Estudos conduzidos por Failla, Thakkar e Kim (2009), avaliando a 
bioacessibilidade de oito cultivares de batata-doce de polpa laranja, indicaram uma baixa bioacessibilidade do all-trans- $\beta$-caroteno desses clones quando cozidos, em relação ao observado em cenouras também cozidas. Segundo Burri (2011), no caso da batata-doce, especificamente, a biodisponibilidade dos carotenoides depende da variedade, da cocção e dos métodos de processamento (Equação 1).

\section{EQUAÇÃO 1 - Relação da biodisponibilidade de carotenoides em batatas-doces sob diferentes condições de processamento.}

BATATA-DOCE CRUA < ASSADA < COZIDA / FERVIDA < FRITA

Fonte: Adaptado de Burri (2011).

Estudos relatam que a bioacessibilidade aumenta significativamente na presença de gordura. Uma digestão simulada mostrou que apenas $0,6 \%$ a 3\% dos carotenoides da batata-doce foram micelados, com um aumento de 7\% no teor de carotenoides em alimentos infantis altamente processados (FAlLA et al., 2009; BURRI, 2011).

Haskell et al. (2004), avaliando a equivalência de vitamina A em homens que apresentavam níveis moderados de vitamina A e que foram alimentados com batata-doce biofortificadas, estimaram a taxa de conversão de 13,4 $\mu \mathrm{g}$ de $\beta$-caroteno para 1 ug de retinol. Burri (2011) afirma que, segundo outros autores, pessoas e animais com baixos níveis de vitamina A parecem converter um percentual maior de $\beta$-caroteno em vitamina A. Em estudo realizado por Ribaya-mercado et al. (2000) com crianças mal nutridas, a taxa de conversão de $\beta$-caroteno em vitamina A variou inversamente aos seus níveis iniciais de vitamina A.

Lee etal. (1999), estudando o metabolismo de $\beta$-caroteno em ratos mongóis alimentados com farinha de milho, demonstraram uma alta correlação inversa 
$(R=0,88)$ com as reservas do fígado, com conversão de $3 \mu$ g de $\beta$-caroteno em $1 \mu \mathrm{g}$ de retinol.

Bengtsson, Alminger e Svanberg (2009) observaram uma bioacessibilidade muito maior de $\beta$-caroteno, variando de $0,5 \%$ a $1,1 \%$ na fase micelar sem gordura e de 11\% a 22\% com 2,5\% de gordura. A concentração de $\beta$-caroteno na fase sobrenadante foi ainda maior, de $24 \%$ a $41 \%$ sem gordura e de $28 \%$ a $46 \%$ com gordura adicionada. Concordando com o relatado por Mills et al. (2009), que observaram aumento na absorção de carotenoides em ratos mongóis com deficiência de vitamina A, a partir de uma alimentação com batata-doce biofortificada, com 12\% de gordura adicionada à dieta. Esse estudo também mostrou que a fritura dobrou a eficiência da incorporação dos $\beta$-carotenos pelas micelas. A bioacessibilidade do $\beta$-caroteno de batata-doce pode ser muito baixa (< $1 \%$ ) em dietas sem gorduras. Uma pequena quantidade de gordura pode aumentar de duas a vinte vezes a bioacessibilidade de $\beta$-caroteno em batata-doce. Mesmo assim, apenas $\pm 25 \%$ (11\% a 48\%) do $\beta$-caroteno em batata-doce estão bioacessíveis e, assim, disponíveis para serem absorvidos pelo intestino (BURRI, 2011).

Dessa forma, um dos desafios para a indústria alimentícia é o de obter produtos sensorialmente aceitáveis, preservando os nutrientes de interesse, assim como compreender e promover uma alta biodisponibilidade e bioacessibilidade dos nutrientes-alvo, para que os ganhos nutricionais sejam potencializados (SEVERO et al., 2021).

\section{Considerações finais}

A concentração de $\beta$-caroteno em alimentos é bastante variável, mostrando-se mais acentuada em cultivares de polpa alaranjada e produtos elaborados com BDB. De forma geral, conforme descrito por vários autores, o processamento durante a elaboração de produtos alimentícios pode resultar em 
redução nos teores de carotenoides, que se alteram de acordo com o modo de processamento. O desenvolvimento de produtos alimentícios com altos teores de $\beta$-caroteno, que apresentem estabilidade em diferentes tipos de processamento e tenham boa aceitação sensorial, consiste em uma alternativa viável para inserção da BDB na alimentação de crianças, com vistas ao combate à hipovitaminose A. No entanto, questões como bioacessibilidade e biodisponibilidade também devem ser levadas em consideração.

\section{Agradecimentos}

À FAPERGS e ao IF Farroupilha pelo incentivo à pesquisa.

\section{Referências}

ANDRADE JR, V. C. et al. Características produtivas e qualitativas de ramas e raízes de batatadoce. Horticultura Brasileira, v. 30, n. 4, p. 584-589, 2012. Disponível em: https://www.scielo.br/ scielo.php?script=sci_arttext\&pid=S0102-05362012000400004\#: :text=A\%20batata\%2Ddoce\%20 (Ipomoea\%20batatas,e\%20baixo\%20custo\%20de\%20produ\%C3\%A7\%C3\%A3o . Acesso em: 10 mar. 2021.

AZEVEDO, S. M. et al. Desempenho agronômico e parâmetros genéticos em genótipos de batata-doce. Horticultura Brasileira, v. 33, n. 1, p. 84-90, mar. 2015. Disponível em: https://doi.org/10.1590/ S0102-053620150000100014. Acesso em: 10 mar. 2021.

BENGTSSON, A.; ALMINGER, M. L.; SVANBERG, U. In vitro bioaccessibility of beta-carotene from heat-processed orange-fleshed sweet potato. Journal of Agriculture and Food Chemistry, v. 57 , p. 9693-8, 2009. Disponível em: https://doi.org/10.1021/jf901692r. Acesso em 11 mar. 2021.

BIOFORT. Disponível em: http://biofort.com.br/. Acesso em 23 ago. 2021.

BOUIS, H. E; SALTZMAN, A. Improving nutrition through biofortification: A review of evidence from HarvestPlus, 2003 through 2016. Glob Food Sec, v.12, p. 49-58, 2017. Disponível em: https:// pubmed.ncbi.nlm.nih.gov/28580239/. Acesso em 23 jun. 2021.

BOVELL-BENJAMIN, A. C. Sweet Potato: A Review of its past, present, and future role in human nutrition. Advances in food and Nutrition Research, v. 52, p. 1-59, 2007. Disponível em: https:// doi.org/10.1016/s1043-4526(06)52001-7. Acesso em: 11 mar. 2021.

BURRI, B. J. Evaluating Sweet Potato as an Intervention Food to Prevent Vitamin A Deficiency. Comprehensive Reviews in Food Science and Food Safety, v. 10, 2011. Disponível em: https:// onlinelibrary.wiley.com/doi/full/10.1111/j.1541-4337.2010.00146.x. Acesso em: 11 mar. 2021. 
CARDOSO, A.D. et al. Avaliação de clones de batata-doce em Vitória da Conquista. Horticultura Brasileira, Brasília, v. 23, n. 4, p. 911-914, out-dez 2005. Disponível em: https://www.scielo.br/scielo. php?script=sci_arttext\&pid=S0102-05362005000400009\&lng=pt\&tlng=pt. Acesso em: 11 mar. 2021.

CAVALCANTE, J. T. et al. Análise de trilha em caracteres de rendimento de clones de batatadoce (Ipomoea batatas). Acta Scientiarum Agronomy, v. 28, p. 261266, 2006. Disponível em: https:// doi.org/10.4025/actasciagron.v28i2.1119. Acesso em: 11 mar. 2021.

CORREIA, L. F. M; FARAONI, A. S; PINHEIRO-SANTANA, H. M. Efeitos do processamento industrial de alimentos sobre a estabilidade de vitaminas. Alimentos e Nutrição, v. 19, n. 1, p. 83-95, 2008. Disponível em: http://serv-bib.fcfar.unesp.br/seer/index.php/alimentos/article/viewFile/204/209? \#: :text=ALIMENTOS\%20SOBRE\%20A\%20ESTABILIDADE\%20DE\%20VITAMINAS,-Laura\%20Fernandes\%20Melo\&text=\%E2\%88\%8ERESUMO\%3A\%200\%20processamento\%20industrial,entre\%20 os\%20constituintes\%20de\%20alimentos . Acesso em: 11 mar. 2021.

COSTA, N. M. B; ROSA, C. O. B. (Org). Alimentos Funcionais. 1. Ed. Rio de Janeiro: Rubio, 2010. 536p. EMBRAPA. Soluções Tecnológicas - Batata-doce Beauregard. Disponível em: https://www.embrapa. br/busca-de-solucoes-tecnologicas/-/produto-servico/7172/batata-doce-beauregard. Acesso em: 19 mar. 2021.

FAILLA, M. L; THAKKAR, S. K.; KIM, J.Y. In vitro bioaccessibility of beta-carotene in orange-fleshed sweet potato (Ipomoea batatas, Lam.). Journal of Agriculture and Food Chemistry, v. 57, p. 10922-27, 2009. Disponível em: https://doi.org/10.1021/jf900415g. Acesso em: 11 mar. 2021.

FAO. The State of Food Insecurity in the World 2014. Strengthening the Enabling Environment for Food Security and Nutrition. Rome: FAO. Disponivel em: http://www.fao.org/3/a-i4030e.pdf. Acesso em 3 set. 2018.

Faostat. FAO statistics division. Disponível em: http://faostat.fao.org/. Acesso em 16 jun. 2021.

FERNANDES, F.R. et al. Biofortificação: batata-doce Beauregard. Embrapa Hortaliças, Brasília, 2014. Disponível em: https://www.embrapa.br/busca-de-publicacoes/-/publicacao/1016136/ biofortificacao-batata-doce-beauregard. Acesso em: 01 dez. 2018.

FIGUEIREDO, J. A. et al. Avaliação de silagens de ramas de batatadoce. Horticultura Brasileira, v. 30, n. 4, 2012. Disponível em: https://www.scielo.br/scielo.php?script=sci_arttext\&pid=S0102-05362012000400024\&lng=pt\&tlng=pt. Acesso em: 13 mar. 2021..

HASKELL M. J. et al. Daily consumption of Indian spinach (Basella alba) or sweet potatoes has a positive effect on total-body vitamin A stores in Bangladeshi men. The American Journal of Clinical Nutrition, v. 80, n. 3, p. 705-714, 2004. Disponível em: https://doi.org/10.1093/ajcn/80.3.705. Acesso em: 22 mar. 2021.

HOTZ, C. et al. A Large-Scale Intervention to Introduce Orange Sweet Potato in Rural Mozambique Increases Vitamin A Intakes among Children and Women. British Journal of Nutrition, v. 108, p. 163-176, 2012. Disponível em: https://doi.org/10.1017/s0007114511005174 . Acesso em: 13 mar. 2021. 
HOTZ, C. et al. Introduction of Beta-Carotene-Rich Orange Sweet Potato in Rural Uganda Results in Increased Vitamin A Intakes among Children and Women and Improved Vitamin A Status Among Children. The Journal of Nutrition, v. 142, n. 10, p. 1871-1880. 2012. Disponível em: https://doi. org/10.3945/jn.111.151829. Acesso em: 13 mar. 2021.

IFPRI. International Food Policy Research Institute. The Challenge of Hunger 2007: Global Hunger Index: Facts, Determinants, and Trends. Washington, D.C., 2014. Disponível em: https://www.ifpri. org/publication/2007-global-hunger-index-challenge-hunger. Acesso em: 15 mar. 2021.

IMDAD, A. et al. Vitamin A Supplementation for Preventing Morbidity and Mortality in Children from 6 Months to 5 Years of Age. Cochrane Database of Systematic Reviews v. 12, n. CD008524, 2010. Disponível em: https://doi.org/10.1002/14651858.cd008524.pub3. Acesso em: 15 mar. 2021.

LAURIE, S. et al. Biofortification of sweet potato for food and nutrition security in South Africa. Food Research International, v. 76, p. 962-970, 2015. Disponível em:https://www.researchgate.net/ deref/http\%3A\%2F\%2Fdx.doi.org\%2F10.1016\%2Fj.foodres.2015.06.001 . Acesso em: 15 mar. 2021.

LEE C. M. et al. Review of animal models in carotenoid research. Journal of Nutritional, v. 129, n. 12 , p. 2271-7. 1999. Disponível em: https://doi.org/10.1093/jn/129.12.2271. Acesso em: 15 mar. 2021.

LIMA D. B.; DAMIANI L. P.; FUJIMORI E. Vitamin a deficiency in brazilian children and associated variables. Revista Paulista de Pediatria, v. 36, p. 176-185, 2018. Disponível em: https://www.scielo. br/scielo.php?script=sci_arttext\&pid=S0103-05822018000200176\&lng=pt\&tlng=pt . Acesso em: 15 mar. 2021.

MILLS, J. P. et al. Sweet potato beta-carotene bioefficacy is enhanced by dietary fat and not reduced by soluble fiber intake in Mongolian gerbils. The Journal of Nutrition, v. 139, p. 44-50, 2009. Disponível em: https://doi.org/10.3945/jn.108.098947 . Acesso em: 15 mar. 2021.

NASCIMENTO, K. O. et al. Caracterização química e informação nutricional de fécula de batata-doce (Ipomoea batatas L.) orgânica e biofortificada. Revista Verde de Agroecologia e Desenvolvimento Sustentável, v. 8, n. 1, p. 132-138, 2013. Disponivel em: https://www.gvaa.com.br/revista/index. php/RVADS/article/view/1774 . Acesso em: 15 mar. 2021.

NESTEL, P. et al. Biofortification of staple food crops. The Journal of Nutrition, v. 136, n. 4, p. 10641067, 2006. Disponível em: https://academic.oup.com/jn/article/136/4/1064/4664193 . Acesso em: 15 mar. 2021.

PLETSCH, L. B. H. et al. Elaboração de balas de batata-doce biofortificada. In: Congresso Brasileiro de Ciência e Tecnologia de Alimentos XXV. Anais do Congresso Brasileiro de Ciência e Tecnologia de Alimentos, Gramado-RS, 2016. p. 1-6. Disponível em: http://www.ufrgs.br/sbctars-eventos/ xxvcbcta/anais/. Acesso em: 15 mar. 2021.

REMONATO, J. R. et al. Qualidade de macarrão tipo talharim elaborado com farinha de batata-doce Beauregard. Revista Agropecuária Técnica, v. 38, n. 2, p. 91-95, 2017. Disponível em: https://doi. org/10.25066/agrotec.v38i2.28647. Acesso em: 15 mar. 2021.

RODRIGUEZ-AMAYA, D. B. Changes in carotenoids during processing and storage of foods. Archivos Latinoamericanos de Nutricion, v. 49, n. 3, p. 38-47, 1999. Disponível em: https://pubmed.ncbi. nlm.nih.gov/10971842/. Acesso em: 15 mar. 2021. 
RODRIGUEZ-AMAYA, D. B. et al. Updated Brazilian data base on food carotenoids: factors affecting carotenoid composition. Journal of Food Composition and Analysis, v. 21, n. 6, p. 445-463, 2008. Disponível em: https://doi.org/10.1016/j.jfca.2008.04.001 . Acesso em: 15 mar. 2021.

RIBAYA-MERCADO J. D. et al. Bioconversion of plant carotenoids to vitamin A in Filipino school-aged children varies inversely with vitamin A status. The American Journal of Clinical Nutrition, v. 72, p. 455-65, 2000. Disponivel em: https://doi.org/10.1093/ajcn/72.2.455. Acesso em: 15 mar. 2021.

SALTZMAN, A. et al. Biofortification Techniques to Improve Food Security. Reference Module in Food Science, v. 1, p. 1-9, 2016. Disponível em: http://dx.doi.org/10.1016/B978-0-08-100596-5.03078-X . Acesso em: 25 jun. 2021.

SANTOS, F. N. et al. Cozimento altera os teores de carotenoides e antocianinas em batatas-doce de polpa alaranjada e roxa. In: Simpósio de Educação e Ciências III. Anais III Simpósio de Educação e Ciências, Jaguari,2018. p. 17-21. CD-ROM. Disponível em: http://conferencias.iffarroupilha.edu. br/index.php/jaguari/IIISEC/index. Acesso em: 21 mar. 2021.

SANTOS, F. N. et al. Elaboration of food products with biofortified sweet potatoes: characterization and sensory acceptability. Revista Chilena de Nutrición, v. 48, n. 1, p. 59-66, 2021. Disponível em: < https://scielo.conicyt.cl/scielo.php?script=sci_arttext\&pid=S0717-75182021000100059\&lng=en \&nrm=iso\&tlng=en >. Acesso em: 16 mar. 2021.

SEVERO et al. Biofortified sweet potatoes as a tool to combat vitamin A deficiency: Effect of food processing in carotenoid contente. Revista Chilena de Nutrición, v. 48, n. 3, p. 414-424, 2021. Disponível em: < http://dx.doi.org/10.4067/S0717-75182021000300414 >. Acesso em: 16 jun. 2021.

SPOLIDORO, J. V. N. Nutrição parenteral em Pediatria. Jornal de Pediatria (Rio J), v. 76 (Supl.3): s339-s48, 2000. Disponível em: < http://www.jped.com.br/conteudo/00-76-S339/port.asp >.

SUAREZ, M. H. et al. Application of multidimensional scaling technique to differentiate sweet potato (Ipomoea batatas (L.) Lam) cultivars according to their chemical composition. Journal of Food Composition and Analysis, v. 46, p. 43-49, 2016. Disponível em: < https://doi.org/10.1016/j. jfca.2015.10.008 >. Acesso em: 16 mar. 2021.

TAN-TORRES, E. T. et al. Cost Effectiveness Analysis of Strategies for Child Health in Developing Countries. British Medical Journal, v. 331, p.1177, 2005. Disponível em: < https://doi.org/10.1136/ bmj.38652.550278.7C >. Acesso em: 16 mar. 2021.

VAN JAARSVELD, P. J. et al. Retention of $\beta$-carotene in boiled, mashed orange-fleshed sweet potato. Journal of Food Composition and Analysis, v. 19, n. 4, p. 321-329, 2006. Disponível em: < http:// www.ask-force.org/web/Africa-Harvest-Sorghum-Lit-1/Jaarsveld-Betacarotene-Sweetpotato-2006. pdf. Acesso em: 16 mar. 2021.

VEDA S. et al. Determination of bioaccessibility of $\beta$-carotene in vegetables by in vitro methods. Molecular Nutrition and Food Research, v. 50, p. 1047 - 1052, 2006. Disponível em: < https://www. researchgate.net/profile/Krishnapura-Srinivasan/publication/6741955_Determination_of_bioaccessibility_of_carotene_in_vegetables_by_in_vitro_methods/links/5abefac90f7e9bfc0459blea/ Determination-of-bioaccessibility-of-carotene-in-vegetables-by-in-vitro-methods.pdf >. Acesso em: 22 mar. 2021. 
VIANA, D. J. S. et al. Potencial de silagens de ramas de batatadoce para alimentação animal. Ciência Rural, v. 41, p. 1466-1471, 2011. Disponível em: <https://www.scielo.br/scielo.php?script=sci_ arttext\&pid=S0103-84782011000800027\&lng=pt\&tlng=pt >. Acesso em: 16 mar. 2021.

VIZZOTTO, M. etal. Composição mineral em genótipos de batata-doce de polpas coloridas e adequação de consumo para grupos de risco. Brazilian Journal of Food Technology, v. 21, p. 1-8, 2018. Disponível em: https://www.scielo.br/scielo.php?script=sci_arttext\&pid=S1981-67232018000100415\&lng=pt \&tlng=pt. Acesso em: 16 mar. 2021.

VIZZOTTO, M. et al. Physicochemical and antioxidant capacity analysis of colored sweet potato genotypes: in natura and thermally processed. Ciência Rural, v. 47, n. 4, p. 1-8, 2017. Disponível em: https://doi.org/10.1590/0103-8478cr20151385. Acesso em: 16 mar. 2021.

World Health Organization (WHO). Micronutrient deficiences: vitamin A deficiency. Disponível em: https://www.who.int/news-room/fact-sheets/detail/malnutrition. Acesso em: 10 mar. 2021.

ZHU, C. et al. Transgenic strategies for the nutritional enhancement of plants. Trends in Plant Science, v. 12, n. 12, p. 548-555, 2007. Disponível em: https://doi.org/10.1016/j.tplants.2007.09.007. Acesso em: 16 mar. 2021. 